The experiential impact of hospitalisation: Parent's accounts of caring for young people with early psychosis

\author{
Authors \\ Gareth Hickman ${ }^{1,2}$ \\ Elizabeth Newton ${ }^{1,2}$ \\ Kelly Fenton ${ }^{1,3}$ \\ Jessica Thompson ${ }^{1,4}$ \\ Zoë VR Boden ${ }^{1}$ \\ Michael Larkin ${ }^{1}$ \\ ${ }^{1}$ University of Birmingham \\ ${ }^{2}$ Coventry and Warwickshire Partnership Trust \\ ${ }^{3}$ Birmingham and Solihull Mental Health Foundation Trust \\ ${ }^{4}$ St Andrew's Healthcare, Birmingham
}

Corresponding author: Dr Michael Larkin, email: m.larkin@bham.co.uk, Address: Department of Psychology, University of Birmingham, Edgbaston, Birmingham, B15 2TT, UK. 


\begin{abstract}
This research examines the experiential impact of hospitalisation on the parents of young people with early psychosis. In-depth interviews were conducted with a small sample of parents, and the resulting transcripts were analysed using Interpretative Phenomenological Analysis (IPA). Five themes emerged from the data: Accepting and blaming, Feeling out of control, Hospitalisation as temporary containment, Feeling let down by services, and Stigma. Aspects of the hospitalisation process were characterised by parents as generally negative, but a number of positive affirmations were also offered regarding the containing, supportive and crucial role of services. Parents' perceptions of hospitalisation as a difficult, and sometimes distressing, experience are exacerbated by the complexity of being the carer of a young person. Negotiating services and boundaries within the context of this relationship contributes to feelings of exclusion and disregard by professionals and services. The implications of this study resonate with the current government mental health strategy with regard to how services can engage and include carers in the mental health system, and equip and enable them to support their relatives with early psychosis.
\end{abstract}


Psychiatric hospitalisation can be distressing, even traumatising (see Berry, Ford, Jellicoe-Jones \& Haddock, 2013), but little is known about the impact of hospitalisation in early psychosis, and even less is known about the perspective of the service-users' family, despite their important role in the care of the young person with psychosis (Addington \& Burnett, 2004). It is estimated that approximately one per cent of the population of the UK will receive a diagnosis of schizophrenia (WHO, 2001), and it is widely understood that schizophrenia and psychotic illnesses develop in late adolescence and early adulthood (Harrop \& Trower, 2001). Early Intervention Services (EIS) have therefore developed within this context. EIS see young people between the ages of 16 and 35 with early psychosis, the aim being to provide sustained multi-disciplinary intervention during the 'critical period' of elevated risk of developing longterm illness (e.g. Birchwood, 1999). Treatment is aimed at promoting recovery in three areas: symptomatic, social, and psychological. Central to an EIS approach therefore is aiming to prevent hospitalisation where possible (Lester, 2004), however hospitalisation does still happen, and for some people hospital is their first encounter with mental health services.

\section{Psychosis and the family}

Currently there are few studies that address the impact of hospitalisation on the service-user's family. A number of studies have focused on the dynamic interactions between relatives/caregivers and individuals with schizophrenia. Research themes that are common in this area include overwhelming feelings (e.g. Saunders \& Byrne, 2002), guilt (Fortune, Smith, \& Garvey, 2005), burden (Awad \& Voruganti, 2008), and coping strategies (Martens \& Addington 2001). Much of this research originates from a positivist epistemology, and less is understood about the lived experiences of these people.

A number of qualitative studies have explored the process of a person developing and living with schizophrenia, and in doing so have indirectly touched upon how family members have experienced the hospitalisation of their relative. Barker, Lavender and Morant (2001) developed a temporal model of 
schizophrenia that included events around the first hospital admission. They identify containment, hope for the future, and compassionate professionals as positive aspects of the first admission, however, mostly parents reported negative experiences, including not being listened to, being offered no choices, perceived professional incompetence, feeling blamed and accused, having no coherent explanations, disagreements with professionals, and having poor or limited understanding of schizophrenia. Whilst such studies offer valuable insights into family members' experience of their relative's hospitalisation, they do not directly examine its experiential impact.

Crisanti (2000) offers a notable exception by examining mothers' experiences of the involuntary hospitalisation of their adult-children with schizophrenia. Crisanti's analysis draws on phenomenology and is idiographic in its use of a small, purposive sample of three mothers. Major themes include experiencing the process as demeaning, feeling baffled, feeling victimised by mental health professionals, and feeling judged as a poor mother. This study represents an important contribution to knowledge of the family perspective, and the current research aims to build on this.

Understanding service-users' and their families' experiences is central to modern health care provision, and recent NICE guidance on psychosis and schizophrenia (2014) includes new measures to support and include carers. The Government strategy "No Health Without Mental Health" (2011) emphasises the need to support, value and include carers in order to improve recovery outcomes for those with mental illhealth. Consequently, the current research aims to better inform our understanding of the experiential impact of hospitalisation on the parents of young people with early psychosis, leading to recommendations that may improve the experience of hospitalisation for relatives and service-users. 


\section{Method}

\section{Context}

This study represents one aspect of a larger 'multiple-perspective design' (Larkin, Shaw \& Flowers, 2013) project that examined three different perspectives of hospitalisation for early psychosis. Other members of the research team explored service-users' (Fenton et al., 2014) and inpatient staff's (Thompson et al., under review) experiences. All three studies were conducted in one NHS Trust, in the Midlands, UK, which has three EIS teams and two inpatient hospitals. Once completed, findings were fed into a service-development project with approximately 150 service-users, inpatient and community staff, managers, and relatives (Larkin, Boden \& Newton, in press).

\section{Participants}

Ethical approval was granted by the local NHS board. Six parents of service-users who had been hospitalised with early psychosis and were currently under the care of a Midlands EIS were recruited via those services (see Table 1). Inclusion criteria required participants to be either living with, or providing care or support to the young person at the time of the hospitalisation.

\section{[TABLE 1 NEAR HERE]}

\section{Data-collection}

Written consent was obtained before participation in the study. Semi-structured interviews focused on collecting participants' narratives of their experiences of their son or daughter's hospitalisation. Questions were open-ended and enquired about the participants' experiences and sense-making, and were specifically situated within the context of their experience. One, approximately 90 minute, 
phenomenological interview was conducted with each participant in their home. Interviews were conducted by the first author, a Clinical Psychology trainee, who was not employed by the EIS or hospital. Interviews were transcribed verbatim and anonymised.

\section{Data analysis}

Interpretative Phenomenological Analysis (IPA) (Smith, Flowers \& Larkin, 2009) was selected because it enables understanding of participants' subjective experiences. It has a contextual-constructivist epistemology and is informed by phenomenological theory (see Smith et al., 2009). The four-stage procedure involved a) reading and re-reading the transcript; the focus being on 'slowing down' and entering the world of the participant; b) initial noting, with a focus on developing descriptive, linguistic and conceptual codes; c) developing emergent themes through re-organisation of the data, and d) searching for connections between emergent themes to develop a thematic structure. The credibility and trustworthiness of the analytic process and the emergent themes was enhanced through keeping a reflexive diary, and discussing emergent themes with other team members throughout the analysis. This process of triangulation and validity checking is considered to enhance the credibility of the interpretation and final analysis.

\section{Results}

Five themes emerged from the data. These were: Accepting and blaming; Feeling out of control; Hospitalisation as temporary containment; Feeling let down by services; and Stigma.

\section{Accepting and blaming}


Participants attempted to make sense of the psychosis by taking a polarised position of either 'acceptance' or 'blame'.

Where participants adopted an understanding and non-blaming position, they appeared more positive towards the young person, could separate the person from the psychosis, and normalised behaviours they considered to potentially contribute to psychoses, such as substance use.

"I think all kids experiment with drugs these days, and a lot of them get away with it. Particularly with cannabis, and the strong stuff, some people are allergic to it as you know. And, erm... it... it brings this thing [psychosis] on." (Joan)

In contrast, a number of participants appeared to adopt a more critical perspective toward the young person, blaming them for developing psychosis:

"It's all self-inflicted I think. I could be wrong. He has an addictive personality I think." (Phillip)

Parents who took this standpoint shared an understanding of the psychosis as being related to external factors, such as substance use, but were less likely to justify or normalise that behaviour. Parents with active difficulty accepting their relative's experience were at the extreme end of this position. The use of dismissive language and doubt in the following quotation is indicative of this, evoking perceptions of malingering.

"When he was in hospital he was talking about these voices that he was hearing and these images that he was seeing. And I was like, 'well, where'd you get that from? This is the first I've heard of it.'... I was just like, you know, gobsmacked and very dismissive of it, 'don't be so bloody stupid'”. (Claire) 
The position that participants adopted toward the young person and the psychosis appears to play a role in forming their subsequent experiences of the hospitalisation as a place of safety and containment, or as a place to hide.

\section{Feeling out of control}

Participants indicated that the pre-hospitalisation phase was an incredibly distressing time. Early psychosis is characterised by its unprecedented nature, and with little or no existing knowledge or experience to draw on, participants felt out of control, overwhelmed and helpless. Here, Phillip reflects on how he and his wife were unsure of how to respond to a progressive deterioration of their son's mental health:

“we just thought there's something wrong with his mental state, and we kept pleading with him

to go and see a doctor but he wouldn't. He kept saying "there's nothing wrong with me". The frustration. You don't know what to do."

Some participants engaged in proactive problem-solving, often taking practical action, such as reading material about psychosis, perhaps as one way to manage feeling out of control. Joan described planning out how she would involve the Early Intervention Team, while Maggie, on feeling that the doctor was not taking her son's psychosis seriously, "took three months off initially [...] to be at home with him".

Efforts to regain a sense of control over difficult situations can be observed throughout participants' accounts. However, even following a period of stabilisation in hospital, participants continued to feel unprepared. The unpredictability of the unknown, how the psychosis might progress, and what the future might hold left participants feeling a continued lack of control. They also spoke of feeling inadequately supported: 
"The lack of support for the carer in the immediate days could definitely be better because it's totally bewildering. I know for people whose family member may have had a condition for a long time, they'll be more familiar with everything, but there's always going to be that stage when it all starts to begin [...] I do feel that there needs to be more support for the carer when it's all happening. " (Sarah)

In the pre-hospitalisation phase, parents feel out of control and in crisis, however even by discharge, they remain unprepared for what is likely to happen and feel unsupported.

\section{Hospitalisation as temporary containment}

Participants' accounts of hospitalisation framed it overwhelmingly as an appropriate intervention that brought relief and respite. The young person was understood to be physically contained, with access to appropriate treatment, and hospital was seen as a place of safety, for self and society. Hospitalisation also offered some psychological containment for the parents' distress, although it came at the cost of feeling blamed by the young person.

All participants reported a sense of relief, which arose in response to a number of mechanisms. Joan expressed how her son was desperately in need of treatment and when doctors agreed to section him she felt "absolutely relieved." Feelings of relief were also engendered by the safety that admission provided. Martin expressed his fear that his son might, through his disinhibited behaviour, place himself at risk in the community, and the safety of the hospital contained these anxieties.

"The Mental Health Hospital is quite secure actually. So I was quite happy that he was there because it felt safe for us really. And that was a big fear, because you know, anything could happen to him, you know? When he's not thinking straight he could say something to the wrong person or people and get hurt!" (Martin) 
Claire's understanding of her son's hospitalisation in terms of possible malingering provided the context through which she made sense of the role of the hospital. Whilst she experienced difficulty reconciling the extent of her son's illness and alludes to him 'hiding' in hospital, she still derived relief from the notion that hospital provided him safety and security.

"My understanding was that he was in a lot of trouble in the outside world and tucked away in there it meant that nobody could get at him, so I was quite relieved actually." (Claire)

A number of participants identified hospitalisation as providing a relief from the burden of caring. For example, Martin suggests he received respite from the stress of caring for his son:

“[It was] The best place for us as well you know, because he's such really hard work when he's out when he's ill, so it gave us a bit of time as well to bloody de-stress sort of thing." (Martin)

Whilst parents appear to experience the hospitalisation as an appropriate means of containment, it was frequently accompanied by the distress of feeling blamed by their child. Inevitably for young people involuntarily hospitalised, the event was experienced from a fundamentally different perspective. Throughout participants' accounts there was the perception that the young person blamed the parent as the cause of their distress, and this was difficult for parents to reconcile with their belief that they were 'doing the best' for them:

"He had this great hatred of me, whatever it was, so it was very difficult for me, it was a great hate. And I think it stemmed from I was the one, I actually put him into the hospital." (Joan)

Thus, hospitalisation provided safety, respite and containment, but was also the source of tension between the parent and young person, because of the parents perceived, or actual, role in the admission.

\section{Feeling let down by services}


Feeling let down by inadequate service provision was the most starkly recorded and pervasive aspect of parents' experiences of hospitalisation. Predominantly this was characterised by negative perceptions of services and by feeling disregarded and under-valued.

Feeling disregarded was engendered through a number of exclusionary practices, particularly the limited information provided to parents on the basis of confidentiality regulations. Almost all participants experienced this as distressing.

“you phone up to ask how's your son and they can't give you any information at all. They were very limited. They'd say "he's just the same, he's the same as he was" you know what I mean? So they wouldn't give hardly anything. I don't think it's on really, is it? ' (Martin)

Parents also perceived hospitals as excluding them through the lack of coherent explanations they offered, particularly regarding diagnosis, treatment and side-effects:

"What would have been nice would have been for someone to sit us down and explain what was wrong with him, you know what I mean? Because they didn't really say anything about anything." (Martin)

This exclusionary disregard ultimately led parents to feeling unvalued, as Sarah describes:

"It's difficult because he's an adult, there were certain things that he didn't want me to be privy to, and yet I'm essentially his carer and I'm his mother, and I know him better than anybody and I feel I should have been consulted more"

Parents' negative experiences appear to be further compounded by their perceptions of professional incompetence. For example, during the process of enacting the Mental Health Act, at the point of the initial hospitalisation, Martin noted: 
"The social worker as well was absolute rubbish. She just didn't know what she was doing. [...] Just, at a very stressful time, just incapable of doing her job."

Similarly, Phillip felt let down by his son's wait to be assessed after admission:

"He didn't get seen by a doctor until - it might have been the Tuesday [...] so that didn't seem too well organised. If you've got to go into a mental health hospital don't go in at a weekend, because there's no staff there!"

Upon discharge, perceptions of support were mixed. Some parents felt services continued to provide inadequate support:

"The Early Intervention team did say they were going to step it up and have family meetings and explain all this and explain all that, but nothing. [...] It's really frustrating at the least. If they say they're gonna do something they should do it." (Martin)

However, others described post-discharge support by agencies external to the hospital, including EIS, positively. Joan felt EIS had "always been there for me." and similarly, Maggie described their EIS worker as "an absolute rock" and "very, very supportive."

Throughout participants' narratives the confusing and bewildering nature of services was a recurrent negative. Parents' difficulty navigating the system is likely to be exacerbated by it being their first experience of hospitalisation. Services overwhelm relatives with too many people and too little explanation:

"When I was at the hospital talking to the intervention team, you've got all these people coming at you. [...] it's like, 'so who do I actually speak to about this?'. 'Do I ring you?' Having so many people is very unhelpful." (Claire) 
Parents' experience of hospitalisation is distressing and bewildering because they are left feeling disregarded and confused about their child's care. Initial perceptions of feeling contained by hospitalisation appear to subside as parents feel increasingly excluded.

\section{Stigma}

Participants' perceived services and hospitalisation as stigmatising, but also suggested that society at large had negative attitudes toward mental health. The nature and practices of services were experienced as stigmatising and embarrassing, for example Joan describes the moment an ambulance indiscreetly came to transport her son to hospital:

"When the ambulance came they were wandering around the village because they couldn't find us where we were, and I actually ran out after them, and I did find that quite [pause] you know [pause], it just happens to be this area you know with the house names. And then obviously someone came out and said "oh Joan, what's the matter?”, you know, and I just kind of ignored it and carried on." (Joan)

A number of accounts alluded to the lack of societal understanding around mental health, and the negative perceptions that are commonplace: “they think 'oh he's just lazy'” (Sarah).

It was notable that parents described how their concern was greater for their child than themselves.

Martin: "obviously there was the embarrassment of a mental illness and then for him to go back to college after it, if he'd have been acting weird that's what I was worried about and going back to work as well."

Interviewer: "You were worried for him?"

Martin: “Oh, yeah, not for me, I'm not embarrassed about it at all. But for Ian himself, because I know he feels that way" 
Evidently stigma is distressing not only by those who live with mental ill health but also for those who support them. Ostensibly, services themselves have some way to go to eradicating stigmatising elements from their practice.

\section{Discussion}

This research explored the impact of hospitalisation on the parents of young people experiencing early psychosis. Many aspects of the hospitalisation were characterised as negative, however it is important not to underestimate the relief, sense of safety and containment that hospitalisation also provided to parents during this time.

Perhaps understandably, parents spoke in depth about feeling out of control during the pre-hospitalisation phase of the illness. This finding is not highlighted in the existing literature, and is likely to reflect the unique experience of parents witnessing their son's or daughter's early psychosis. A lack of precedent is likely to exacerbate feelings of distress, which helps explain why hospitalisation was welcomed. This seems to be compounded the lack of information received. In contrast to existing literature, themes of guilt, self-blame, shame and failure (e.g. Jungbauer, Wittmund, Dietrich \& Angermeyer, 2004) were absent in the current study. Instead, parents mainly viewed hospitalisation as an appropriate strategy, and in accordance with Barker et al. (2001) they welcomed it for the containment and relief it offered. This might be understood in terms of a return to control that parents experienced through believing their child was safe and being treated. Although this stands in stark contrast to the views of service-users (Fenton et al., 2014) and inpatient staff (Thompson et al., under review) that are reported in the complementary studies that formed our team's multiple-perspective research project, who both describe the inpatient unit as threatening, non-therapeutic and chaotic. 
The negative experiences identified in this study support existing literature. Feeling disregarded was common to all participants, and as also identified by Barker et al. (2001) who reported parents 'not being listened to' and 'having no coherent explanations'. The experience of services as 'bewildering and overwhelming' echoes the findings of Crisanti (2000), who notes parents felt baffled by the hospitalisation process. Additionally, parents in the current study referred to the stigmatising potential of psychosis and hospitalisation; this is in-keeping with the considerable literature in this area (see Van Zelst, 2009).

The position adopted by parents in relation to the young person's psychosis requires further exploration. Some parents' belief that their son was responsible for the development of his psychosis, and/or that the psychosis was not a legitimate illness, seemingly influenced their experiences of hospitalisation. It has been argued that critical relatives, high in expressed emotion (EE), are more likely to blame the serviceuser for their psychosis and that this increases relapse rates and other negative outcomes (see Barrowclough \& Hooley, 2003). Some experiences in this study could be read as idiographic examples of elevated EE, and it seems these parents are currently given little opportunity to adjust their (critical) position to one that could be more helpful to the young person. Family intervention (FI) for psychosis has sought to address such difficulties and reduce distress within families. The current research indicated a range of difficulties (family communication, affective expression, conflict reduction, stigma, social support, and negotiating the mental health system) that could benefit from need-determined FI. Fadden (2009) recomends offering help as early as possible; families are particularly willing to engage in times of crisis, such as hospital admissions. NICE guidance (2014) suggests FI should be offered routinely, and can begin during inpatient care. This study suggests this is also the time when parents currently feel most confused and excluded.

\section{Clinical implications}


This study points to a number of positive affirmations of the supportive and crucial role that hospital, EIS, and voluntary services provide in the context of early psychosis, and it is the responsibility of services to build upon these. However, the study also raises a number of concerns regarding hospitalisation. The mental health outcomes strategy, No health without mental health (2011), offers a relevant strategic framework for addressing a number of these challenges. This includes The Triangle of Care - Carers included: A guide to best practice in acute mental health care, which sets out six key elements of good practice for mental health professionals working with carers. This study suggests the following are particularly relevant: "Staff are 'carer aware' and trained in carer engagement strategies"; "Policy and practice protocols on confidentiality and sharing information are in place"; and "A range of carer support services is available”.

Looking across our team's multiple-perspective project, service-users (Larkin et al., in press) viewed maintaining relationships with family during hospitalisation as important for their recovery, however, inpatient staff (Thompson et al., under review) failed to mentioned carers at all, although they often saw themselves in pseudo-parental roles. This suggests staff acknowledge these young people's critical developmental stage, but do not currently consider carers during hospitalisation. These findings highlight the importance of training hospital staff in carer engagement, with a particular focus on the containing influence staff can have during the initial stages of the admission, and also with regards to inclusion, communication and information sharing. Similarly, this study highlights the importance of developing practice protocols on confidentiality and information sharing with relatives. Contracting involved individuals regarding how and what will be disclosed may be one way to address this. Individualised contracts can reduce carers' feelings of disregard and exclusion, provide clearly defined respectful boundaries, and increase flexibility in information sharing. 
The carer's need for information must be balanced with the service-user's right to privacy (Szmukler \& Bloch, 1997) and professionals may struggle with the possibility of causing harm through a failure to disclose versus respecting the service-users' wishes. Slade et al. (2007) have proposed a framework for best clinical practice, which makes an important distinction between 'general information', which can always be shared without consent, and 'personal information', which is new to the carer and where consent needs to be considered. This framework presents a decisional-based model regarding what to share, where the central mediating factor is clinical judgement. The current research supports the introduction of such frameworks. Finally, this study supports the need for identified staff members as a point of contact and source of information in order to reduce parents feeling overwhelmed and intimidated by the large number of professionals involved in services. Additionally, identified staff members will encourage reciprocity and dialogue between services and families.

The present study utilises a small and purposeful sample, consistent with IPA guidelines, and further research is required. However, the inclusion of fathers, both living with and separated from the young person's mother, adds to the available literature. Siblings' and spouses' perspectives are also underexplored. The current sample consists only of parents of sons, and whilst the sample accessed is thought to represent the local demographic, it is limited by the lack of Black and Minority Ethnic participants.

\section{Summary}

Parents in this study found hospitalisation for early psychosis both containing and a source of relief primarily because they felt their child was safe and accessing treatment. Additionally though, parents found hospitalisation confusing, excluding, and stigmatising. 


\section{Acknowledgements}

Thanks to Terry Twomey, Janet Kenworthy-Harvey, Donna Luck and all of the participants in both the research and service-development project. 


\section{Acknowledgements}

Thanks to Terry Twomey, Janet Kenworthy-Harvey, Donna Luck and all of the participants in both the research and service-development project. 


\section{References}

Addington, J., \& Burnett, P. (2004). Working with families in the early stages of psychosis. In J. Gleeson \& P.D. McGorry (Eds.), Psychological interventions in early psychosis: A treatment handbook (pp.99-116). Chichester, UK: Wiley \& Sons Ltd.

Awad, G., \& Voruganti, L.N.P. (2008). The burden of schizophrenia on caregivers: A review. Pharmacoeconomics, 26, 149-162.

Barker, S., Lavender, T., \& Morant, N. (2001). Client and family narratives on schizophrenia. Journal of Mental Health. 10, 199-212.

Berry, K., Ford, S., Jellicoe-Jones, L., \& Haddock, G. (2013). PTSD symptoms associated with the experiences of psychosis and hospitalisation: A review of the literature. Clinical Psychology Review, 33, 526-538.

Birchwood, M. (1999). Early intervention in psychosis: The critical period. In P.D. McGorry \& H.J. Jackson (Eds.), The recognition and management of early psychosis. A preventative approach (pp. 226-264). Cambridge: Cambridge University Press.

Barrowclough, C. \& Hooley, J.M. (2003). Attributions and expressed emotion: A review. Clinical Psychology Review, 23, 849-880.

Crisanti, A.S. (2000). Experiences with involuntary hospitalization: A qualitative study of mothers of adult children with schizophrenia. Schizophrenia Research, 45, 79-81.

Department of Health (2011). No health without mental health: A cross-government mental health outcomes strategy for people of all ages. London: Department of Health.

Fadden, G. (2009). Family interventions in psychosis. In H. Beinart, P. Kennedy \& S. Llewelyn (Eds.), Clinical Psychology in Practice (pp.199-208). Chichester: Wiley-Blackwell. 
Fenton, K., Larkin, M., Boden, Z.V.R., Thompson, J., Hickman, G., \& Newton, E. (2014). The experiential impact of hospitalisation in early psychosis: Service-user accounts of inpatient environments. Health and Place, 30, 234-241.

Fortune, D.G., Smith, J.V., \& Garvey, K. (2005). Perceptions of psychosis, coping, appraisals, and psychological distress in the relatives of patients with schizophrenia: An exploration using selfregulation theory. British Journal of Clinical Psychology. 44, 319-331.

Harrop, C., and Trower, P. (2001). Why does schizophrenia develop at late adolescence? Clinical Psychology Review, 21, 241-265

Jungbauer, J., Wittmund, B., Dietrich, S., \& Angermeyer, M.C. (2004). The disregarded caregivers: Subjective burden in spouses of schizophrenia patients. Schizophrenia Bulletin, 30, 665-675.

Larkin, M., Boden, Z.V.R. \& Newton, E. (in press). On the Brink of Genuinely Collaborative Care: Reflections on the Use of Experience-based Co-design for Translating Qualitative Research into Service Development. Qualitative Health Research (special issue on 'Knowledge Translation').

Lester, H. (2004). Early intervention: An idea whose time has come? Research Policy and Planning. 22, $71-78$

Martens, L., \& Addington, J. (2001). The psychological well-being of family members of individuals with schizophrenia. Social Psychiatry and Psychiatric Epidemiology, 36, 128-133.

National Institute for Clinical Excellence (2014). Psychosis and schizophrenia in adults: Treatment and Management (NICE clinical guidance 178). London: National Institute for Health and Care Excellence.

Reid, K., Flowers, P., \& Larkin, M. (2005). Exploring lived experience. The Psychologist, 18, 20-23.

Saunders, J.C., \& Byrne, M.M. (2002). A thematic analysis of families living with schizophrenia. Archives of Psychiatric Nursing, 5, 217-223. 
Slade, M., Pinfold, V., Rapaport, J., Bellringer, S., Banerjee, S., Kuipers, E., \& Huxley, P. (2007). Best practice when service users do not consent to sharing information with carers. British Journal of Psychiatry. 190, 148-155.

Smith, J.A., Flowers, P., \& Larkin, M. (2009). Interpretative Phenomenological Analysis: Theory, Method and Research. London: Sage.

Szmukler, G.I., \& Bloch, S. (1997). Family involvement in the care of people with psychoses. An ethical argument. British Journal of Psychiatry, 171, 401-405.

Thompson, J., Boden, Z.V.R., Larkin, M., Hickman, G., Fenton, K., \& Newton, E. (under review). The Experiential Impact of Hospitalisation in Early Psychosis: Staff Accounts of Working with Young People.

Van Zelst, C. (2009). Stigmatization as an environmental risk in schizophrenia: A user perspective. Schizophrenia Bulletin, 35, 2, 293-296.

WHO (2001). World health report: Mental health, new understandings, new hope. Geneva: World Health Organisation. 
Table 1: Participant demographics

\begin{tabular}{|c|c|c|c|c|}
\hline Pseudonym & $\begin{array}{l}\text { Relative } \\
\text { (pseudonym) }\end{array}$ & Relationship & $\begin{array}{l}\text { Age of young } \\
\text { person } \\
\text { (at first } \\
\text { hospitalisation) }\end{array}$ & Carer circumstances \\
\hline Joan & James & Mother - Son & $21(19)$ & $\begin{array}{l}\text { Employed part-time / Full time } \\
\text { carer }\end{array}$ \\
\hline Sarah & Chris & Mother - Son & $25(23)$ & $\begin{array}{l}\text { Employed full-time / Full time } \\
\text { carer }\end{array}$ \\
\hline Claire & Shaun & Mother-Son & $22(20)$ & $\begin{array}{l}\text { Employed part-time / Limited } \\
\text { contact with son until } \\
\text { hospitalisation / Now full time } \\
\text { carer }\end{array}$ \\
\hline Martin & Ian & Father - Son & $18(16)$ & Unemployed / Part-time carer \\
\hline Maggie & George & Mother-Son & $20(18)$ & $\begin{array}{l}\text { Employed full-time / Full-time } \\
\text { carer }\end{array}$ \\
\hline Phillip & William & Father - Son & $21(18)$ & Retired / Previously full-time \\
\hline
\end{tabular}




\begin{tabular}{|l|l|l|l|}
\hline & & & carer, currently sporadic care \\
\hline
\end{tabular}

